\title{
Effect of Sacubitril/Valsartan on Neurocognitive Function: Current Status and Future Directions
}

\author{
Jason Galo ${ }^{1} \mathbb{D} \cdot$ Diego Celli $^{1} \cdot$ Rosario Colombo $^{2}$ \\ Accepted: 1 October 2020 / Published online: 16 October 2020 \\ C) Springer Nature Switzerland AG 2020
}

\begin{abstract}
Sacubitril/valsartan is a new medication approved for the treatment of heart failure with reduced ejection fraction. While the drug failed to meet the primary endpoint in patients with heart failure with preserved ejection fraction in the PARAGON-HF trial, improvements were noted in several secondary endpoints. Valsartan is an angiotensin receptor blocker and sacubitril is a neprilysin inhibitor. Neprilysin is postulated to have a role in the degradation of beta-amyloid in the brain; therefore, sacubitril could theoretically increase beta-amyloid plaque deposition in the brain and potentially increase the risk of Alzheimer's disease. Although pre-clinical and clinical studies have shown promising safety results, those studies have been heavily criticized for short monitoring time and targeted populations. In accordance with the requirements of the US Food and drug Administration (FDA), the ongoing Prospective Evaluation of Cognitive Function in Heart Failure: Efficacy and Safety of Entresto compared to Valsartan on Cognitive Function in Patients with Chronic Heart Failure and Preserved Ejection Fraction (PERSPECTIVE; NCT02884206) multicenter, randomized, double-blinded trial is assessing the long-term neurocognitive effects and safety of sacubitril/valsartan, and results are expected in early 2022.
\end{abstract}

\section{Neprilysin and Alzheimer's Disease}

Sacubitril, a component of Entresto, is a neprilysin inhibitor that is used in combination with valsartan in the treatment of heart failure with reduced ejection fraction (HFrEF). Neprily$\sin$ is postulated to have a role in the degradation of beta-amyloid in the brain, and the accumulation of beta-amyloid plaques is pathognomonic of Alzheimer's disease [1,2]. Several studies have shown that polymorphisms in the genes encoding for the neprilysin enzyme are associated with Alzheimer's disease. By inhibiting neprilysin, sacubitril may theoretically lead to increased levels of beta-amyloid, raising the concern that physicians may need to carefully monitor their patients on Entresto for potential neurocognitive side effects.

Jason Galo

jason.galo@jhsmiami.org

1 Division of Internal Medicine, Department of Medicine, University of Miami/Jackson Memorial Hospital, 1611 NW 12th Ave, Central Building 600D, Miami, FL 33136, USA

2 Division of Cardiovascular Disease, Jackson Memorial Hospital, 1801 NW 9th Ave, Suite \#209, Miami, FL 33136, USA

\section{Key Points}

Sacubitril/valsartan is a new medication for the treatment of heart failure with reduced ejection fraction; it has been hypothesized that the long-term use of sacubitril, a neprilysin inhibitor, might be associated with increased risk for Alzheimer's disease.

Pre-clinical and clinical studies have shown promising safety results, but have been criticized due to the short period of follow-up and inclusion of healthy, young volunteers.

The Prospective Evaluation of Cognitive Function in Heart Failure: Efficacy and Safety of Entresto compared to Valsartan on Cognitive Function in Patients with Chronic Heart Failure and Preserved Ejection Fraction (PERSPECTIVE) trial is assessing the long-term neurocognitive safety of sacubitril/valsartan, and results are expected in 2022. 


\section{Preclinical Data}

A study from 2017 evaluated the theoretical risk of beta-amyloid accumulation following long-term neprilysin inhibition, given the known concerns about Entresto. In vitro testing showed that neprilysin and its active metabolite sacubrilat is selective for the human neprilysin gene, which is responsible for degrading various physiologically relevant substrates such as atrial natriuretic peptide, C-type natriuretic peptide, and B-type natriuretic peptide, as well as beta-amyloid. Preclinical studies focused on the short-term effects of sacubitril/valsartan on the clearance of beta-amyloid from the cerebrospinal fluid (CSF) of cynomolgus monkeys, as well as concentrations of beta-amyloid in the CSF, brain, and plasma of human volunteers. A study that evaluated the long-term effects of Entresto in beta-amyloid deposition in the brain of cynomolgus monkeys was conducted over 39 weeks. CSF concentrations of sacubrilat, the active neprilysin inhibitor, reached similar levels in these non-human primates compared to human healthy volunteers from other studies. The CSF levels of sacubrilat were enough to inhibit neprilysin in the cynomolgus monkeys. In a shorter 16-day study, CSF concentrations of aggregable beta-amyloid were increased due to a decreased CSF clearance rate. However, after 39 weeks, there was no evidence of sacubitril/valsartan-related microscopic brain changes or beta-amyloid deposition in brain tissue of the cynomolgus monkeys [3]. Although these are promising results, it is not clear if the long-term exposure of the human brain and CSF to sacubitril/valsartan could cause alterations in beta-amyloid levels sufficient to cause beta-amyloid deposition.

\section{US FDA Approval and the PERSPECTIVE Trial}

Sacubitril/valsartan was approved for clinical use in HFrEF based on the PARADIGM-HF trial, but in its approval letter the US Food and Drug Administration (FDA) asked for a follow-up trial [the Prospective Evaluation of Cognitive Function in Heart Failure: Efficacy and Safety of Entresto compared to Valsartan on Cognitive Function in Patients with Chronic Heart Failure and Preserved Ejection Fraction (PERSPECTIVE) trial; NCT02884206] to clarify the long-term neurocognitive safety of neprilysin inhibition by sacubitril. By statute, the FDA required Novartis to conduct a clinical trial (rather than a nonclinical or observational study) to identify "unexpected serious risks of cognitive dysfunction" with the use of Entresto (sacubitril/valsartan). Based on appropriate scientific data, the FDA specifically asked for a "multicentered, randomized, double-blind, and active-controlled trial to evaluate the effects of Entresto compared to valsartan on cognitive function," using a "comprehensive neurocognitive battery and PET imaging" in patients with chronic heart failure with preserved ejection fraction (HFpEF) [4].

\section{Further Studies}

There are limited case reports documenting the development of psychiatric symptoms and neurocognitive deficits, including one with otherwise unexplainable intracranial volume loss in the setting of recent initiation of sacubitril/ valsartan [5]. A small retrospective cohort study comprising 102 patients followed up for 3 months found no differences in cognitive performance using a Mini-Mental State Examination between heart failure patients receiving Entresto and previous conventional therapy [6].

A neurocognitive sub-study of the PARADIGM-HF trial did not find any dementia-related adverse effects of sacubitril/valsartan, but the trial was terminated early and was of relatively short duration (27 months). Furthermore, a follow-up of the PARADIGM-HF study found no increase in adverse events secondary to dementia after 4.25 years in the study participants [2]. A 2016 central nervous system (CNS) penetration study of sacubitril/valsartan in young healthy volunteers reported promising but inconclusive results. The study found that sacubitril/valsartan administration was statistically associated with increased levels of non-aggregable beta-amyloid isoforms in the CSF of the human participants, but there was no statistically significant increase in plaqueforming aggregable beta-amyloid isoforms [7]. Furthermore, this study's results may not be applicable to the population that generally will receive Entresto, since they were mostly healthy young volunteers for whom Entresto would be less commonly prescribed, compared to older patients with heart failure for whom Entresto is indicated.

Data from the FDA Adverse Event Reporting System between 2015 and 2017 involving 9004 entries showed that sacubitril/valsartan was not associated with increased odds of reported cognition- and dementia-related adverse effects and was not linked to a disproportionate rate of these events compared to other medications [8].

Interestingly, a recent cohort study examined the relationship between initiation of Entresto and subsequent prevalence of depression in 37 patients awaiting heart transplant. There is a known association between heart failure and depression, as well as an association between depression and cognitive dysfunction. The study found that end-stage heart failure patients who were treated with Entresto had improved symptomatology that correlated with increased 6-min walk test (6MWT) function and maximum oxygen consumption $\left(V \mathrm{O}_{2 \max }\right)$. In parallel, depression, which was measured by the Beck Depression Inventory (BDI) II scale, was less prevalent after 1 year in those patients who received 
Entresto. Ongoing and future studies evaluating the relationship between Entresto and cognitive dysfunction should account for this possible confounder [9].

\section{Sacubitril/Valsartan in Preserved Ejection Fraction Heart Failure (PARAGON-HF Trial)}

Sacubitril/valsartan failed to meet the primary efficacy endpoint in patients with HFpEF, but the drug improved several secondary endpoints. For example, New York Heart Association (NYHA) class improved significantly in the patients who were randomized to take Entresto compared to those randomized to take valsartan. There was also a modest, non-statistically significant lower rate of hospitalizations for heart failure with sacubitril/valsartan than with valsartan. Notably, there were no reported adverse events related to worsening cognition, but these patients were followed only for a total of 29 months [10]. Despite the study not meeting its primary endpoint, it is possible that the drug may still receive FDA approval in the months ahead. It will also be important to assess pre-specified secondary 'neurocognitive endpoints' of the PARAGON-HF trial.

\section{The PERSPECTIVE Trial}

As required by the US FDA, the PERSPECTIVE trial will use a comprehensive battery of tests with an evaluation of longitudinal change of cognitive domains including memory, executive function, and attention involving 592 subjects (Table 1) [11, 12]. Patients with symptomatic chronic heart failure NYHA class II-IV, ejection fraction above $40 \%$, and N-terminal prohormone of brain natriuretic peptide (NT-proBNP) $\geq 125 \mathrm{pg} /$ $\mathrm{mL}$ at the screening visit are being enrolled in this study and are being randomized to receive either Entresto 97/103 mg per os (PO) twice a day (BID) or valsartan $40 \mathrm{mg}$ or $80 \mathrm{mg}$
PO BID for 3 years. The primary outcome measure will be the change from baseline in the CogState Global Cognitive Composite Score (GCCS). The secondary outcome measures include changes in amyloid plaque deposition over time, change from baseline in individual cognitive domains such as memory, executive function, and attention, and change from baseline in the summary score of the Instrumental Activities of Daily Living (IADL) scale. The key exclusion criteria include patients with pre-existing dementia, a mini-mental status exam score $<24$ at screening, and the inability to perform cognitive tests based on a significant motor or sensory deficiency [11]. The PERSPECTIVE trial is expected to provide definitive answers in May 2022. At this time, it remains unclear how the coronavirus disease 2019 (COVID-19) pandemic will impact the recruitment or trial process, but it is possible that a delay in the expected trial timeline may occur.

Although this data will be beneficial for the medical community, this industry-managed clinical trial involving a small number of patients followed for a relatively short period of time could lack enough power to reach definitive conclusions. Since amyloid deposits can continue to form over a long period of time, post-marketing phase IV surveillance and prolonged follow-up of large trials may be pivotal for this analysis.

\section{Conclusion}

In summary, sacubitril, one of the active components of Entresto, is a neprilysin inhibitor whose mechanism of action may lead to unintentional increases of beta-amyloid levels in the brain. Pre-clinical data on the effect of Entresto on beta-amyloid deposition has shown promising safety results. The US FDA approved Entresto with the condition that there is a clinical trial to identify the serious risks of cognitive dysfunction with its use. Further studies, including a neurocognitive sub-study of the PARADIGM trial, have shown no evidence of cognitive-related adverse events in patients who have taken Entresto, although the follow-up

Table 1 Key inclusion and selected exclusion criteria of the PERSPECTIVE Trial, which will enroll 592 participants for its primary outcome measure of change from baseline in the GCCS [11]

\begin{tabular}{|c|c|}
\hline Inclusion criteria & Selected exclusion criteria \\
\hline $\begin{array}{l}\text { Chronic heart failure with current symptoms NYHA class II-IV } \\
\text { Left ventricular ejection fraction }>40 \% \\
\text { NT-pro BNP } \geq 125 \mathrm{pg} / \mathrm{mL} \text { at screening visit } \\
\text { Patient with evidence of adequate functioning to complete study assess- } \\
\text { ments }\end{array}$ & $\begin{array}{l}\text { Patients with a clinical diagnosis of Alzheimer's disease or other } \\
\text { dementia syndromes or any indication for or current treatment with } \\
\text { cholinesterase inhibitors and/or another prescription dementia treat- } \\
\text { ment (i.e., memantine) } \\
\text { Mini-Mental State Examination score less than } 24 \text { at screening } \\
\text { Clinically significant cerebral pathology that may impact cognition as } \\
\text { assessed by central MRI reader } \\
\text { Inability to perform cognitive battery or other study evaluations }\end{array}$ \\
\hline
\end{tabular}

GCCS CogState Global Cognitive Composite Score, MRI magnetic resonance imaging, NT-proBNP N-terminal prohormone of brain natriuretic peptide, NYHA New York Heart Association, PERSPECTIVE Prospective Evaluation of Cognitive Function in Heart Failure: Efficacy and Safety of Entresto compared to Valsartan on Cognitive Function in Patients with Chronic Heart Failure and Preserved Ejection Fraction 
time was relatively short. During the PARAGON-HF trial, Entresto failed to meet its primary efficacy endpoint, but improved a number of secondary endpoints when used in patients with HFpEF. The PERSPECTIVE trial is an ongoing clinical trial that will provide important answers regarding Entresto's safety profile and much needed clarification regarding the potential risk of cognitive side effects.

\section{Declarations}

Disclosures None of the paper's contents have been previously published. All authors have read and approved the manuscript.

Funding There was no funding for this publication.

Conflicts of Interest Jason Galo, Diego Celli, and Rosario Colombo declare that they have no potential conflicts of interest that might be relevant to the contents of this article. There are no conflicts of interest for any author, or ties with industry.

Ethics Approval Not applicable.

Consent to Participate Not applicable.

Consent for publication All authors have reviewed the edits and are in agreement for the publication.

Availability of data and materials Not applicable.

Code Availability Not applicable.

\section{References}

1. Castello MA, Soriano S. On the origin of Alzheimer's disease. Trials and tribulations of the amyloid hypothesis. Ageing Res Rev. 2014;13:10-2.
2. Krittanawong C, Kitai T. The head and the heart: potential long-term side effect of ARNI. J Am Coll Cardiol. 2017;69(14):1879-80.

3. Schoenfeld HA, et al. The effect of angiotensin receptor neprilysin inhibitor, sacubitril/valsartan, on central nervous system amyloidbeta concentrations and clearance in the cynomolgus monkey. Toxicol Appl Pharmacol. 2017;323:53-655.

4. Services, D.o.H.a.H. NDA APPROVAL (New Drug Application) 207620. 2015. https://www.accessdata.fda.gov/drugsatfda_docs/ appletter/2015/207620Orig1s0001tr.pdf. Accessed 2019.

5. Wooster J, Cook EA, Shipman D. Psychiatric manifestations with sacubitril/valsartan: a case report. J Pharm Pract. 2020;33(4):553-7.

6. De Vecchis R, et al. Cognitive performance of patients with chronic heart failure on sacubitril/valsartan: a retrospective cohort study. Herz. 2019;44(6):534-40.

7. Langenickel TH, et al. The effect of LCZ696 (sacubitril/valsartan) on amyloid-beta concentrations in cerebrospinal fluid in healthy subjects. Br J Clin Pharmacol. 2016;81(5):878-90.

8. Perlman A, et al. Cognition- and dementia-related adverse effects with sacubitril-valsartan: analysis of the fda adverse event report system database. J Card Fail. 2018;24(8):533-6.

9. Cacciatore $\mathrm{F}$, et al. Effect of sacubitril-valsartan in reducing depression in patients with advanced heart failure. J Affect Disord. 2020;272:132-7.

10. Solomon SD, et al. Angiotensin-neprilysin inhibition in heart failure with preserved ejection fraction. $\mathrm{N}$ Engl J Med. 2019;381(17):1609-20.

11. National Institutes of Health. Efficacy and safety of LCZ696 compared to valsartan on cognitive function in patients with chronic heart failure and preserved ejection fraction (PERSPECTIVE). 2016. https://clinicaltrials.gov/ct2/show/NCT02884206. Accessed 14 May 2019.

12. Cannon JA, et al. Dementia-related adverse events in PARADIGM-HF and other trials in heart failure with reduced ejection fraction. Eur J Heart Fail. 2017;19(1):129-37. 